\title{
Sequential Delivery of BMP2-Derived Peptide P24 by Thiolated Chitosan/Calcium Carbonate Composite Microspheres Scaffolds for Bone Regeneration
}

\author{
Zhaozhen Wang, ${ }^{1}$ Xujie Liu, ${ }^{2,3}$ Vidmi Taolam Martin, ${ }^{1}$ Mohamed Abdullahi Abdi, ${ }^{1}$ \\ Lijun Chen, ${ }^{4}$ Yong Gong, ${ }^{1}$ Yiran Yan, ${ }^{1}$ Liming Song, ${ }^{1}$ Zhongxun Liu, ${ }^{1}$ Xianliao Zhang, \\ Yan Chen $\left(\mathbb{D},{ }^{4} \text { and Bo Yu }{ }^{1}\right)^{1}$ \\ ${ }^{1}$ Department of Orthopedics, Zhujiang Hospital of Southern Medical University, Guangzhou 510282, China \\ ${ }^{2}$ Graduate School at Shenzhen, Tsinghua University, Shenzhen 518055, China \\ ${ }^{3}$ State Key Laboratory of New Ceramics and Fine Processing, School of Materials Science and Engineering, Tsinghua University, \\ Beijing 100084, China \\ ${ }^{4}$ Department of Ultrasonic Diagnosis, Zhujiang Hospital, Southern Medical University, Guangzhou 510282, China
}

Correspondence should be addressed to Yan Chen; smu_chen@163.com and Bo Yu; gzyubo@163.com

Zhaozhen Wang, Xujie Liu, and Vidmi Taolam Martin contributed equally to this work.

Received 4 April 2020; Accepted 19 May 2020; Published 13 July 2020

Academic Editor: Hui-Qi Xie

Copyright (c) 2020 Zhaozhen Wang et al. This is an open access article distributed under the Creative Commons Attribution License, which permits unrestricted use, distribution, and reproduction in any medium, provided the original work is properly cited.

\begin{abstract}
The combination of tissue-engineered bone scaffolds with osteogenic induction molecules is an important strategy for critical-sized bone defects repair. We synthesized a novel thiolated chitosan/calcium carbonate composite microsphere (TCS-P24/CA) scaffold as a carrier for bone morphogenetic protein 2- (BMP2-) derived peptide P24 and evaluated the release kinetics of P24. The effect of TCS-P24/CA scaffolds on the proliferation and differentiation of bone marrow mesenchymal stem cells (BMSCs) was evaluated by scanning electron microscope (SEM), CCK-8, ALP assay, alizarin red staining, and PCR. A $5 \mathrm{~mm}$ diameter calvarial defect was created, then new bone formation was evaluated by Micro-CT and histological examination at 4 and 8 weeks after operation. We found the sequential release of P24 could last for 29 days. Meanwhile, BMSCs revealed spindleshaped surface morphology, indicating the TCS-P24/CA scaffolds could support cell adhesion and mRNA levels for ALP, Runx2, and COL1a1 were significantly upregulated on TCS-10\%P24/CA scaffold compared with other groups in vitro $(p<0.05)$. Similarly, the BMSCs exhibited a higher ALP activity as well as calcium deposition level on TCS-10\%P24/CA scaffolds compared with other groups $(p<0.05)$. Analysis of in vivo bone formation showed that the TCS-10\%P24/CA scaffold induced more bone formation than TCS-5\%P24/CA, TCS/CA, and control groups. This study demonstrates that the novel TCS-P24/CA scaffolds might contribute to the delivery of BMP2-derived Peptide P24 and is considered to be a potential candidate for repairing bone defects.
\end{abstract}

\section{Introduction}

Regeneration of critical-sized bone defects resulting from tumor resection and congenital malformation or trauma is still a challenging problem in orthopedic surgery [1-3]. Autograft is the "gold standard" and clinical approach for bone defect reconstruction; it has considerable limitations such as insufficient graft material, and unpredictable bone resorption $[4,5]$. Hence, bone tissue engineering has been recommended as a promising approach for bone regeneration $[6,7]$. However, most of the bone tissue engineering scaffolds were recorded for the significant lack of osteoinductivity. The combination of osteoinductive growth factors or peptides is of important significance [8]. 
BMP-2 has been proved previously in inducing bone formation owing to its better osteoinductive activity $[9,10]$. BMP-2 is known as well too costly, and it usually needs to be applied in a high-dose for clinical therapy, followed by side effects such as promotion of tumor angiogenesis and excessive bone resorption [11, 12]. Alternatively, BMP-2 derived peptide $\mathrm{P} 24$ has been investigated and demonstrated as an enhanced effect of osteogenesis [13]. The P24 peptide is composed of small molecules and has chemical stability and structural alignment, which is beneficial to its biological effect [3]. Besides, the P24 peptide contained ample phosphorylated serine and aspartic acid, which can improve the acceleration of nucleation and mineralization [14]. Also, P24 peptide has a low production cost and is suitable for preparation [3]. According to these characteristics, BMP-2derived peptide $\mathrm{p} 24$ seems to have application space.

Over the past few decades, scaffolds used to release peptides or proteins have received considerable attention. Chitosan has attracted remarkable attention for bone tissue engineering owing to its promising nontoxicity, biodegradability, and biocompatibility [15]. The addition of the thiol group on the amino group of chitosan can ameliorate some properties of chitosan [16]. Chitosan is not soluble in neutral medium, however, the thiolated chitosan showed good solubility under the condition of neutral $\mathrm{pH}$ [17]. Therefore, thiolated chitosan-based scaffolds suitable for delivering peptides or proteins sensitive to $\mathrm{pH}$ changes were prepared [18]. This thiol group on thiolated chitosan might be more conducive to the delivery of cysteine-rich peptides or proteins in the thiolated chitosan-based scaffolds.

However, the structure of the pure thiolated chitosan scaffold has the drawback of quick degradation and weak mechanical properties [19]. Therefore, thiolated chitosan scaffolds may be compounded with calcium carbonate, which is bioabsorbable and has good bone conductivity and biocompatibility. Synthetic calcium carbonate composite scaffolds have been considered as a promising material for bone defect repair [20]. In this research, carboxymethyl chitosan known as a derivative of chitosan, with better water solubility, has been used to prepare and incorporate at the same time an intermediate formula for calcium carbonate. The calcium carbonate hybrid microspheres have excellent biocompatibility, bone conductivity, and biodegradability [21]. According to these findings, we believe that when calcium carbonate microsphere, being synthesized by in-situ precipitation method and combined with thiolated chitosan, can be used as an effective carrier of P24 peptide [22-25]. However, thiolated chitosan/calcium carbonate composite scaffolds for P24 peptide sustained release have not been reported yet. Thus, it is highly significant to analyze the P24 sustained release characteristics for the potential application of TCS-P24/CA scaffolds in bone tissue engineering.

In this study, we synthesized a novel TCS-P24/CA scaffolds for the continuous delivery of P24 peptide. First of all, we tested the physical properties of TCS-P24/CA scaffolds in vitro. Secondly, we performed the proliferation and shape of BMSCs on the TCS-P24/CA scaffolds. Thirdly, the ALP activity, osteogenic-specific genes, and calcium deposition of BMSCs cultured on the TCS-P24/CA scaffolds were evaluated. Finally, calvarial bone defect models were created to evaluate the effect of TCS-P24/CA scaffolds for bone regeneration.

\section{Materials and Methods}

Calcium chloride, chitosan (degree of deacetylation: $80 \%-$ 95\%), ammonia carbonate, and sodium tripolyphosphate were purchased from Sinopharm Chemical Reagent Co. Ltd., China. Chondroitin sulfate $\mathrm{C}$ and carboxymethyl chitosan (Molecular weight: $\sim 150 \mathrm{kDa}$, deacetylation degree: $\geq 90 \%$ ) were purchased from Rubio Co. Ltd, Germany. 2iminothiolane hydrochloride was purchased from Sigma, USA. P24 Peptide $(\mathrm{N} \rightarrow \mathrm{C}$ : KIPKA SSVPT ELSAI STLYL SGGC) was synthesized by Shanghai ZiYu Biotech Co. China.

2.1. Thiolated Chitosan Fabrication. Thiolated chitosan (TCS) was fabricated by a previously described method [26]. Briefly, $400 \mathrm{mg}$ of chitosan was dissolved in $200 \mathrm{~mL}$ $1 \%$ acetic acid for $5 \mathrm{~h}$ to obtain a $0.2 \%(w / v)$ chitosan solution. $80 \mathrm{mg}$ of 2-iminothiolane hydrochloride was added into the solution, and the $\mathrm{pH}$ was adjusted to 6 . Furthermore, the mixed solution was dialyzed against $5 \mathrm{mM} \mathrm{HCl}$ once, $5 \mathrm{mM}$ $\mathrm{HCl}$ (containing $1 \% \mathrm{NaCl}$ ) twice, $5 \mathrm{mM} \mathrm{HCl}$ once, and $1 \mathrm{mM} \mathrm{HCl}$ once, 1 day for each time. The TCS samples were obtained by lyophilizing the mixed solution at $-80^{\circ} \mathrm{C}$ and $20 \mathrm{~Pa}$.

2.2. Calcium Carbonate Microspheres (CA) Fabrication. $10 \mathrm{mg} / \mathrm{mL}$ of carboxymethyl chitosan and $0.4 \mathrm{~mol} / \mathrm{L}$ of calcium chloride solution were mixed and stirred for 10 minutes, recorded as solution $\mathrm{A}$. Concerning solution $\mathrm{B}, 20 \mathrm{mg} / \mathrm{mL}$ of chondroitin sulfate and $0.8 \mathrm{~mol} / \mathrm{L}$ of ammonium carbonate solution were mixed and stirred for 10 minutes. Solution B was added drop by drop into solution $\mathrm{A}$ and then stirred for half an hour. Finally, the solution was mixed and washed 4 times, and then the calcium carbonate microspheres were obtained by drying the sediment for 48 hours.

2.3. TCS-P24/CA Scaffold Fabrication. $100 \mathrm{mg}$ calcium carbonate microspheres (CA) were added to $5 \mathrm{~mL} 2 \%$ thiolated chitosan (TCS) solution and mixed by ultrasonic crusher. The different amounts of P24 were used to fabricate TCS/CA, TCS-5\%P24/CA, or TCS-10\%P24/CA mixed solution. Therefore, a 96-well plate was used as a mold and the mixed solution was lyophilized at $-80^{\circ} \mathrm{C}$ and $20 \mathrm{~Pa}$ to obtain the TCS/CA, TCS-5\%P24/CA, or TCS-10\%P24/CA scaffolds.

2.4. In Vitro P24 Release Study from TCS-P24/CA Scaffold. TCS-P24/CA scaffold was prepared as previously described. TCS/CA scaffolds loaded with P24 were placed in Eppendorf tube. After that, $3 \mathrm{~mL}$ PBS was added to the Eppendorf tube. All samples were continuous shaking at $60 \mathrm{rpm}$ and incubated under $37^{\circ} \mathrm{C}$. During the designed time interval, the amount of $0.5 \mathrm{~mL}$ release medium was tested and replaced by the PBS. The concentration of P24 is determined by the BCA detection kit (Nanjing Jiancheng, China) according to the company's guidelines. 
2.5. Cell Preparation. BMSCs were isolated as described elsewhere [27]. The primary BMSCs were cultured in DMEMF12 with $10 \%$ fetal bovine serum in a mixed environment at $37^{\circ} \mathrm{C}$ and $5 \% \mathrm{CO} 2$. Cell passage numbers $\mathrm{P} 3$ and $\mathrm{P} 6$ were identified and utilized for all experiments.

2.6. Characterization of Scaffold and Observation of Cell Morphology. The interior morphology of the TCS-P24/CA composite scaffolds was observed by scanning electron microscopy (SEM) as described [28]. And then, BMSCs were seeded onto TCS-P24/CA scaffolds and cultured in DMEMF12 with $10 \%$ fetal bovine serum. After 5 days of coculture, samples were fixed with $0.25 \%$ glutaraldehyde solution for $24 \mathrm{~h}$ and immersed in $\mathrm{OsO} 4$ for $1 \mathrm{~h}$ and then rinsed 5 times in 1x PBS. The samples were dehydrated with increasing concentrations of acetone (30-100\%). Therefore, the samples were fixed on aluminum stubs and then observed under scanning electron microscopy (Hitachi S-3000 N, Japan).

2.7. Cell Proliferation Assay. BMSCs were seeded on TCSP24/CA scaffolds. At present time points, the Cell Counting Kit-8 (CCK-8, Dojindo) was used to assess cell activity according to the user manual, and OD values were measured at $450 \mathrm{~nm}$ by a microplate reader.

2.8. In Vitro Osteogenesis Study. BMSCs were cultured for 1, 3 , and 7 days. Alkaline phosphatase (ALP) activity of the BMSCs in the different groups was determined by the ALP assay kit (Nanjing Jiancheng, China) according to the user manual. The calcium mineral deposition formed by BMSCs was evaluated by alizarin red staining (AR-S). BMSCs were cultured in osteogenic induction medium for 21 days, cells were fixed in $4 \%$ paraformaldehyde, rinsed three times with PBS and treated with alizarin red (Beyotime) for $10 \mathrm{~min}$, and washed with ultrapure water. Three randomly selected fields were analyzed by Image-J for calcium mineral deposition area under a microscope.

2.9. Real-Time Polymerase Chain Reaction (PCR). The total RNA was isolated by Trizol reagent, and the synthesized cDNA was used to perform PCR. Specific primers (Table 1) were used for PCR amplification to analyze the expression of type I collagen al (COLla1), alkaline phosphatase (ALP), and runt-related transcription factor 2 (Runx2). Transcriptor cDNA Synth Kit and Fast Start Universal SYBR Green Master were used for PCR. All of the reactions were normalized to GAPDH.

2.10. In Vivo Study. All in vivo experiments were conducted according to the protocols approved by the Southern Medical University Institutional Animal Care and Use Committee. Twenty-four female SD rats (180-250 g) were used in this study, and rats were randomly assigned to the control group (no scaffold), TCS/CA group, TCS-5\%P24/CA group, and TCS-10\%P24/CA group. General anesthesia was induced by $1.0 \mathrm{ml} 2 \%$ pentobarbital sodium. Under general anesthesia, a dental bur was used to create a circular calvarial bone defect of $5 \mathrm{~mm}$. Then, the scaffolds were implanted into the calvarial bone defect, and the skin was closed. After 4 and 8 weeks, all calvarial samples were harvested and soaked in $4 \%$ paraformaldehyde.

2.11. Micro-CT Analysis. The harvested calvarial samples for weeks 4 and 8 were examined using an advanced microcomputed tomography instrument (Micro-CT, ZKKS-MCSharp-IV, Zhongke Kaisheng Bio, Inc.) with scanning parameters of a $0.5 \mathrm{~mm}$ aluminum filter, $200 \mathrm{~mA}$, and $50 \mathrm{kV}$. Morphometric parameters such as the bone mineral density (BMD) were analyzed.

2.12. Histological Examination. After Micro-CT imaging, all calvarial samples were fixed and decalcified, and the specimens were dehydrated through an alcohol gradient and embedded in paraffin blocks. $5 \mu \mathrm{m}$ thick serial slices was prepared and subsequently stained with hematoxylin and eosin (HE) and Masson's trichrome staining.

2.13. Statistical Analysis. All data are expressed as mean \pm SD ; the student's $t$-test was used to examine whether the differences between groups were statistically significant. The probability level of significant difference is $p<0.05$.

\section{Results}

3.1. Characterization of Scaffold, Morphology, and Proliferation of BMSCs on Scaffold. The inner structure and morphology of the scaffold were examined by Scanning Electron Microscope (SEM). As shown in Figure 1(a), the majority of the pores have a diameter from $90-120 \mu \mathrm{m}$. To evaluate whether the scaffolds affected cell growth, BMSCs were seeded onto TCS/CA, TCS-5\%P24/CA, and TCS-10\%P24/CA scaffolds. The SEM results indicated that most of the BMSCs showed a spindle morphology on TCS/CA, TCS-5\%P24/CA, and TCS-10\%P24/CA (Figure 1(b): B1, B2, and B3), which have been shown to promote the differentiation and maintain the multipotency efficiency of BMSCs. Cell viability and proliferation were measured by the CCK-8 assay (Figure 2(a)). CCK-8 results showed that the proliferation of BMSCs on TCS-P24/CA scaffolds (Figure 2(a)) was similar to control groups after 1,3,7,10 days. These results demonstrated that the TCS-P24/CA scaffolds were noncytotoxic to BMSCs and might support a good proliferation pattern of BMSCs.

3.2. Release Behaviors of the BMP2-Derived Peptide P24. The cumulative release amounts of P24 from TCS-P24/CA scaffolds are shown in Figure 1(c). The TCS-P24/CA scaffolds could release P24 peptide continuously for 29 days. The cumulative release curve of $\mathrm{P} 24$ peptide showed that during the 29-day release cycle, the release rate of P24 peptide was faster in the first 7 days, then slowed down and the release curve tended to be stable. $80 \%$ of P 24 was released from the TCS-P24/CA scaffold within 29 days. In summary, the TCS-P24/CA scaffold can release P24 peptide continuously and effectively.

3.3. In Vitro Osteogenesis. Osteogenic differentiation by BMSCs within the TCS-P24/CA scaffolds was evaluated by the ALP activity, the expression of osteogenic genes, and the level of calcium deposition. As shown in Figure 2(b), on 
TABle 1: Primer sequences of osteogenic markers.

\begin{tabular}{lcc}
\hline mRNA & Forward $\left(5^{\prime}-3^{\prime}\right)$ & Reverse $\left(5^{\prime}-3^{\prime}\right)$ \\
\hline COL1a1 & GCAACAGTCGCTTCACCTACA & CAATGTCCAAGGGAGCCACAT \\
GAPDH & TGTGTCCGTCGTGGATCTGA & TTGCTGTTGAAGTCGCAGGAG \\
ALP & GGCTGGAGATGGACAAATTCC & CCGAGTGGTAGTCACAATGCC \\
Runx2 & CCAACCCACGAATGCACTATC & TAGTGAGTGGTGGCGGACATAC \\
\hline
\end{tabular}

COL1a1: collagen type I alpha 1; GADPH: glyceraldehyde-3-phosphate dehydrogenase; ALP: alkaline phosphatase; Runx2: runt-related transcription factor 2.

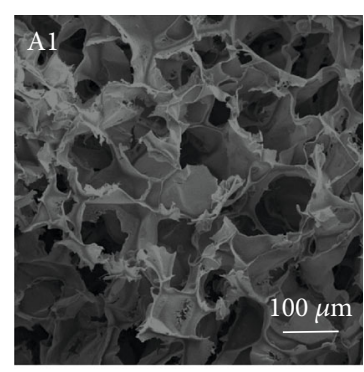

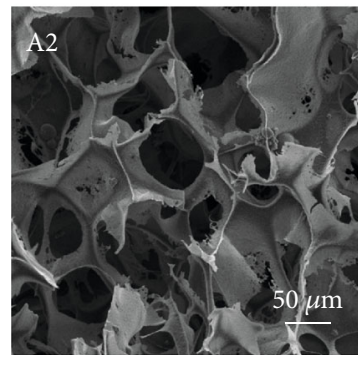

(a)
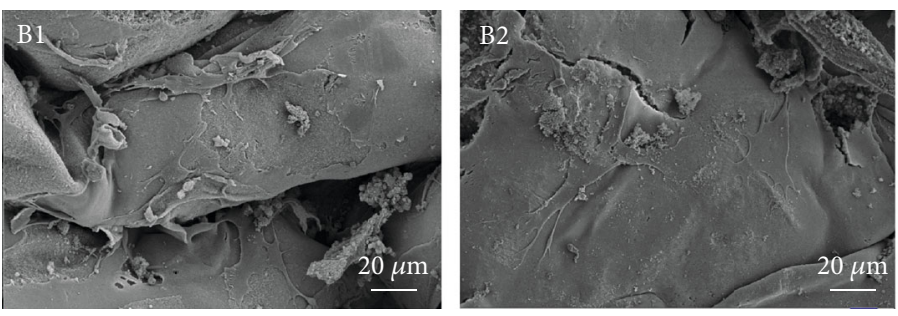

(b)
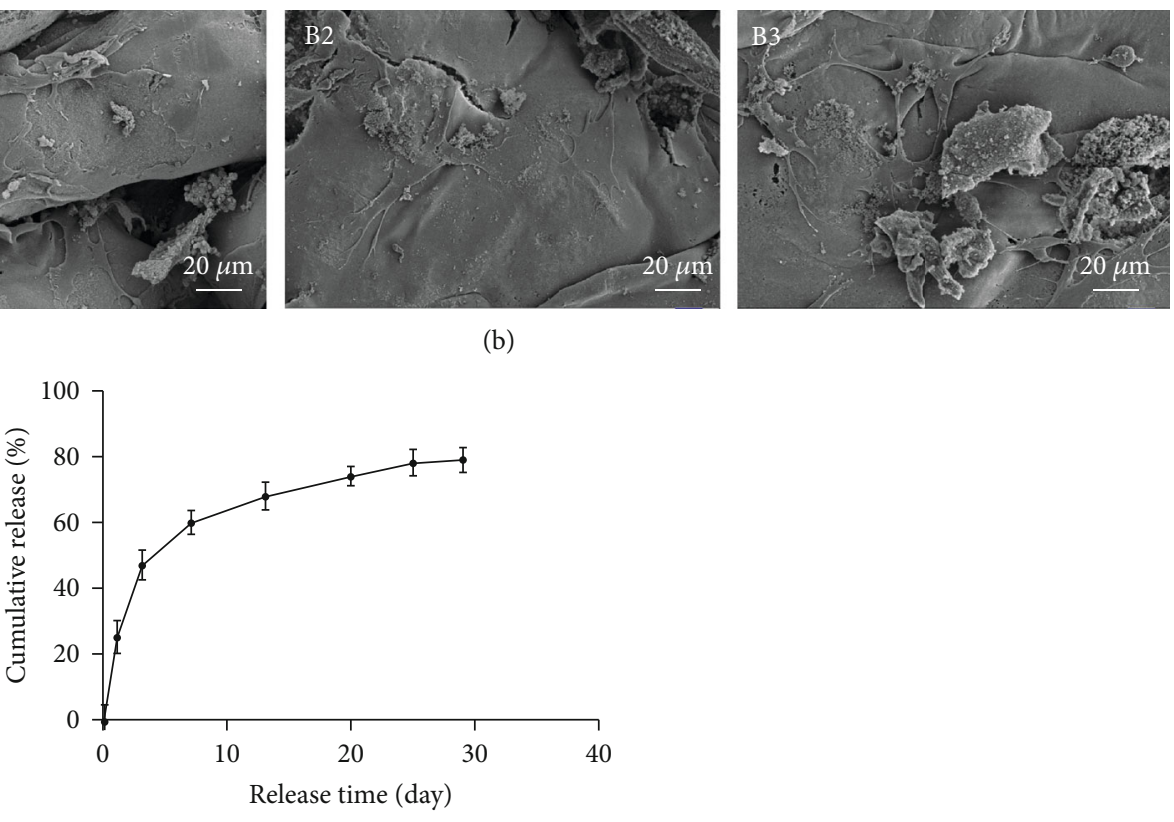

$\rightarrow$ TCS-P24/CA

Figure 1: (a) SEM image of TCS-P24/CA scaffold (A1:100X; A2:200X). (b) SEM view of BMSCs cultured on (B1) TCS/CA, (B2) TCS$5 \% \mathrm{P} 24 / \mathrm{CA}$, and (B3) TCS-10\%P24/CA scaffolds at a magnification of 5000X. (c) Cumulative in vitro release curves of the TCS-P24/CA scaffold. Error bars represent mean $\pm \mathrm{SD}(n=3)$.

day 3, the ALP activity of BMSCs in the TCS-5\%P24/CA and TCS-10\%P24/CA groups was significantly higher than control and TCS/CA groups $(p<0.01)$. On day 7 , the ALP activity of BMSCs of TCS-10\%P24/CA group was significantly higher than other groups $(p<0.05)$. The level of calcium nodule deposition after 21 days in culture was investigated by alizarin red staining. The results revealed that the control group showed almost no positive staining; TCS/CA and TCS$5 \% \mathrm{P} 24 / \mathrm{CA}$ groups showed positive staining, while TCS$10 \% \mathrm{P} 24 / \mathrm{CA}$ group presented the most significantly positive staining $(p<0.05$ Figure $2(\mathrm{c}))$. Moreover, as shown in
Figure 2(d), PCR showed that ALP, Runx2, and COL1al gene expression increased in the TCS-10\%P24/CA group compared to the other groups $(p<0.05)$.

3.4. TCS-P24/CA Scaffolds Promote Bone Regeneration. We next investigated the effect of TCS-P24/CA scaffold on rat calvarial defect repair at 4 and 8 weeks after operation. The Micro-CT results revealed that there was no bone repair in the control group (Figure 3(a)), whereas the TCS/CA and TCS-5\%P24/CA groups demonstrated a small amount of new bone formation into the defect. Additionally, bone 

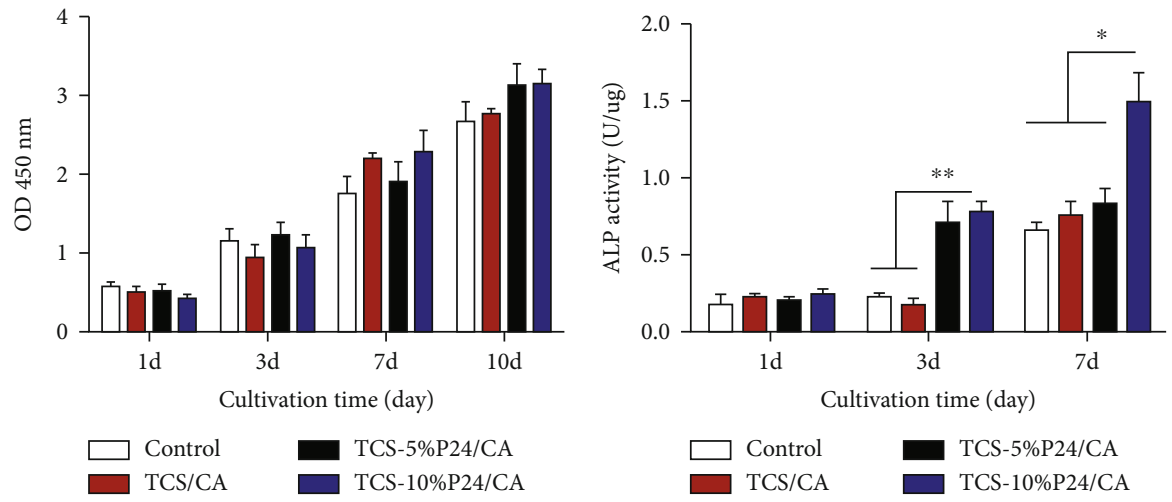

(a)

(b)

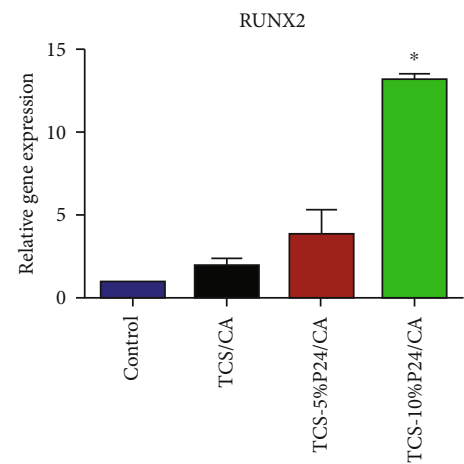


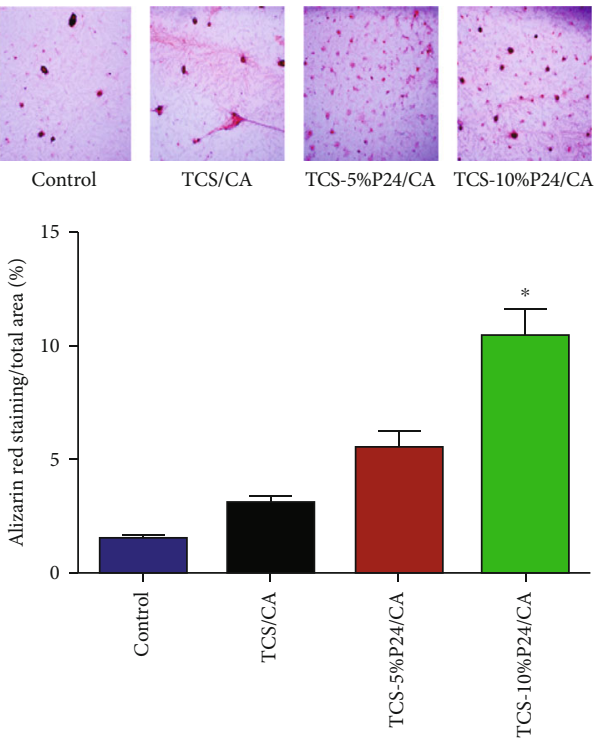

(c)

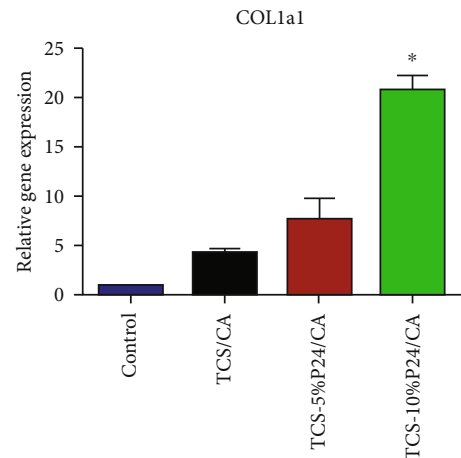

ALP

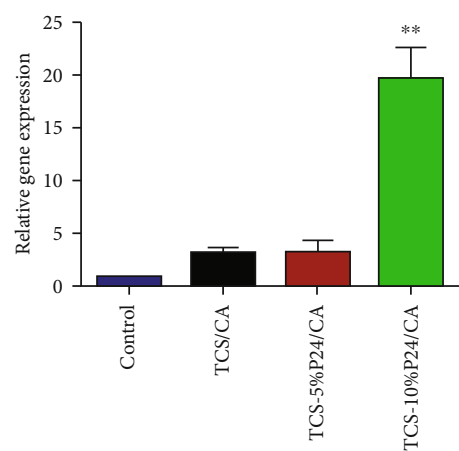

(d)

FIgure 2: (a) CCK-8 test of BMSCs viability after coculture with TCS/CA, TCS-5\%P24/CA, and TCS-10\%P24/CA scaffolds at days 1, 3, 7, and 10. (b) The relative ALP activity of BMSCs cultured in a scaffold-stimulated medium. (c) Alizarin red S staining of BMSCs cultured with TCS/CA, TCS-5\%P24/CA, and TCS-10\%P24/CA scaffolds for 21 days. (d) The level of the mRNA for osteogenic-specific genes (Runx2 and ALP) and related matrix genes (COL1a1) of rat BMSCs cultured on TCS-P24/CA scaffolds for 7 days. Levels, quantified using real-time RT-PCR, are normalized to the reference gene GAPDH. $\left({ }^{*} p<0.05 ;{ }^{* *} p<0.01\right)$; Error bars represent mean \pm SD $(n=3)$. 




(a)

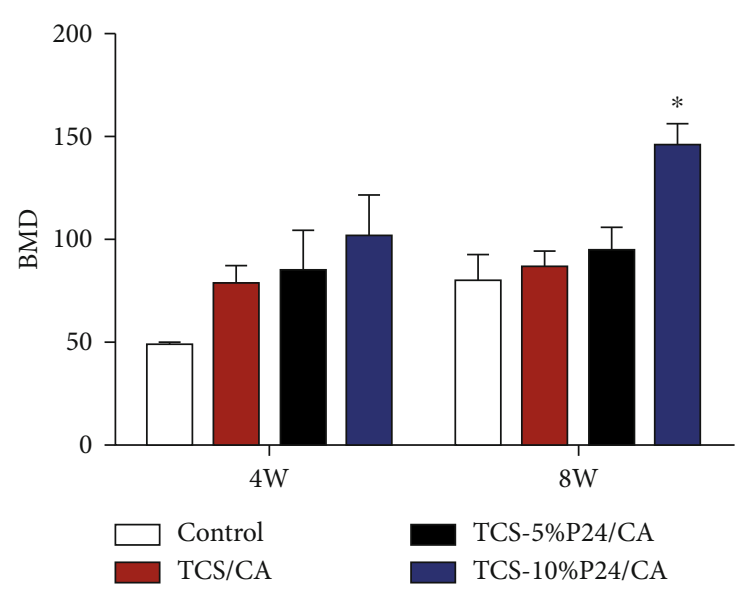

(b)

FIGURE 3: Evaluation of calvarial bone defect repair in vivo. (a) Micro-CT analysis of skulls 4 and 8 weeks post-surgery. (b) Quantitative analysis of BMD 4 and 8 weeks postsurgery. ${ }^{*} p<0.05$.

formation was significantly increased in the TCS$10 \% \mathrm{P} 24 / \mathrm{CA}$ group compared to other groups at 8 weeks. After 8 weeks, according to Micro-CT analysis, the BMD was significantly higher in the TCS-10\%P24/CA group.

We performed $\mathrm{HE}$ and Masson staining to further verify the repair effect after 4 and 8 weeks (Figure 4). At 4 weeks, although there was no new bone formation in the control group, which had fibrous tissue formation, a small amount of new bone formation was found in the TCS/CA, TCS5\%P24/CA, and TCS-10\%P24/CA groups. 8 weeks after implantation, different from the lack of obvious bone formation in the defect area of the control, TCS/CA, and TCS$5 \% \mathrm{P} 24 / \mathrm{CA}$ groups, the repaired area of TCS-10\%P24/CA group had stronger osteogenic ability, and more osteoblasts were observed around the new bone, and vascularization could be seen at the edge of the bone defect. In summary, our results demonstrated that TCS-10\%P24/CA significantly enhances bone regeneration in vivo.

\section{Discussion}

Excellent bone tissue engineering scaffold should not only support cell attachment, cell proliferation, and migration but also could to carry and release osteogenic induction molecules $[29,30]$. In the present study, the TCS-P24/CA scaffold was used to accomplish the controlled delivery of P24 peptide to enhance osteogenesis. Our findings demonstrated the safety and efficiency of the TCS-P24/CA scaffold in bone regeneration and affirmed the potential application value of this material in bone regeneration.
The efficiency of BMP-2 depends on the form of delivery. It has been proved that long-term delivery of BMP-2 is more effective in promoting bone formation in a certain dose range [31]. At present, one of the pivotal issues of BMP-2 is to select a suitable drug delivery system to ensure its controlled release and maintain biological activity. In many cases, the BMP-2 deposited in the material is released with an early eruption. Therefore, a more reliable carrier for sequential delivery of growth factors to the target has received widespread attention. As a carrier for localization and delivery of BMP-2, the system includes polylactic-glycolic acid, absorbable collagen [32]. Chitosan is also a well-tested vehicle. The effectiveness of thiolated chitosan as a drug controlled release scaffold has been confirmed by drugs such as insulin [33]. However, most studies have focused on systematic drug delivery [28]. At the same time, although thiolated chitosan has many advantages in tissue engineering, however, the structure of the pure thiolated chitosan scaffold has the drawback of quick degradation and weak mechanical properties. Therefore, thiolated chitosan scaffolds may be compounded with inorganic minerals to improve the mechanical properties. At present, there are many kinds of bone scaffold materials in tissue engineering technology. Calcium carbonate used in this experiment is a kind of artificial bone, which also includes nanohydroxyapatite, tricalcium phosphate, calcium sulfate, and calcium phosphate cement [34]. As a bone substitute material, nanohydroxyapatite has been used in the clinic for a long time and shows good biocompatibility, bioactivity, and bone conductivity, but it has low bending strength, high brittleness, easy to fracture, and difficult to 

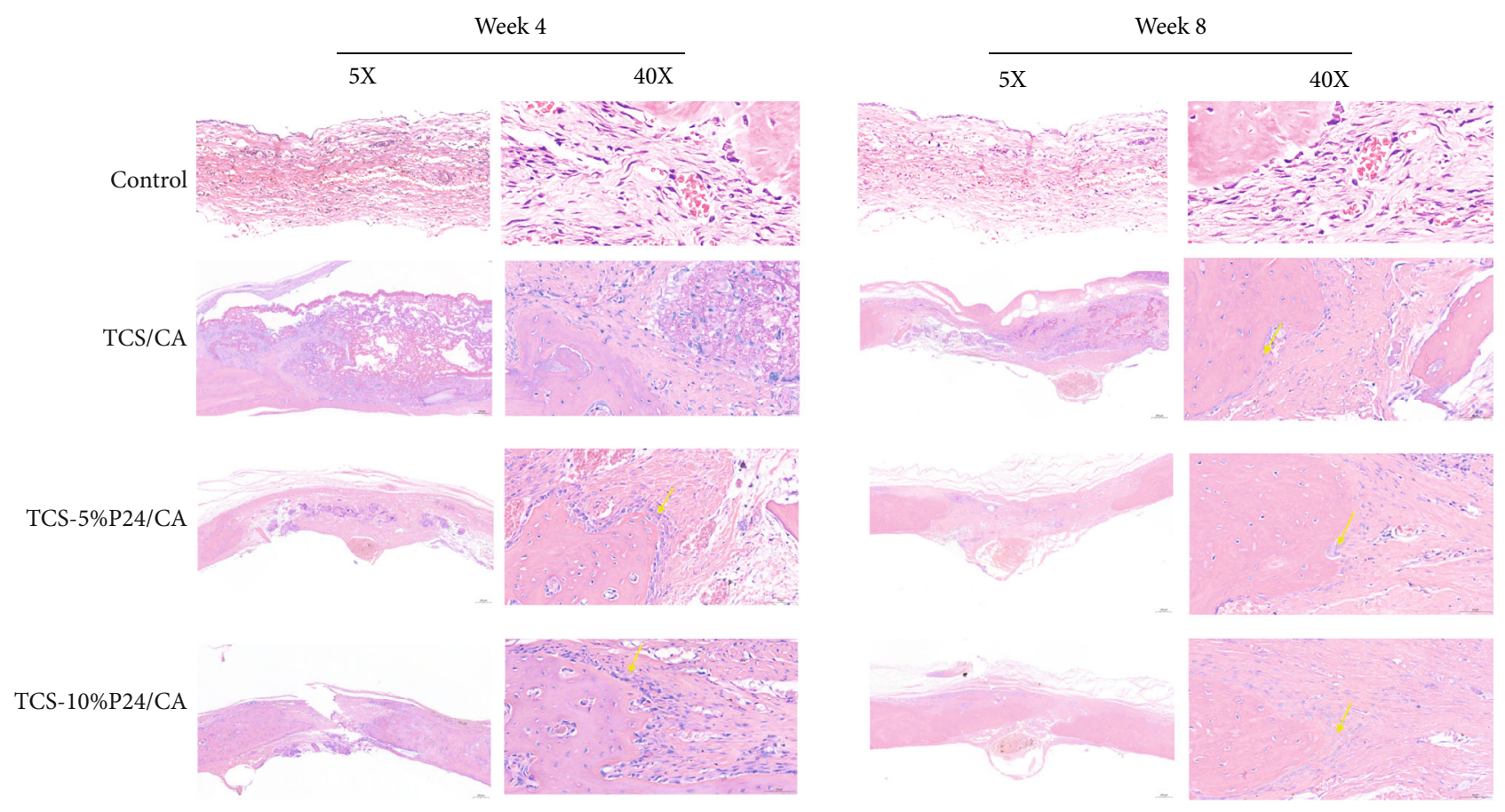

(a)
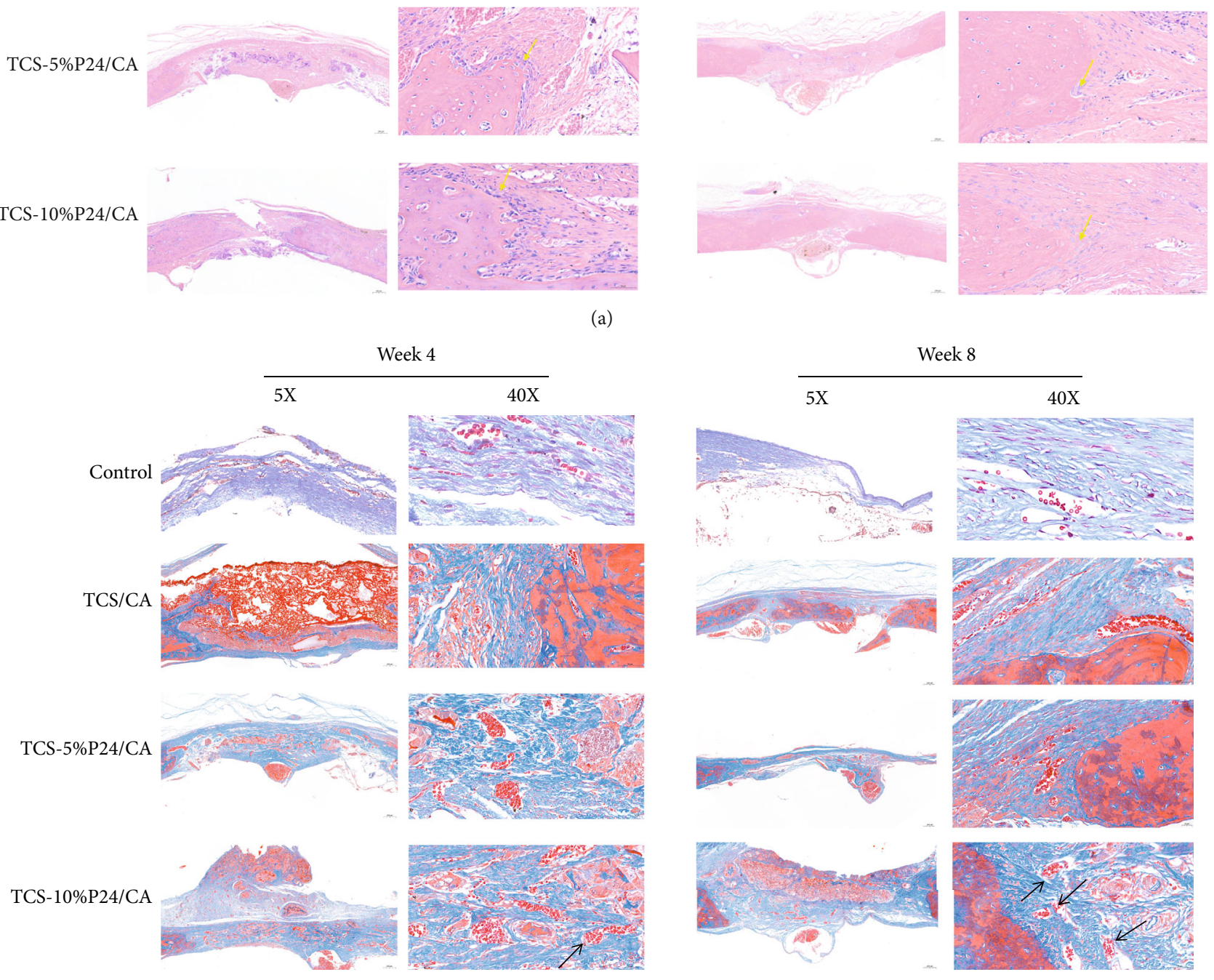

(b)

FIgURE 4: Histology of the cranial specimens at 4 and 8 weeks after implantation in vivo. (a) Hematoxylin and eosin staining of harvested tissues in Control, TCS/CA, TCS-5\%P24/CA, and TCS-10\%P24/CA groups. Yellow arrow represents new bone. (b) Masson's trichrome staining of harvested tissues in Control, TCS/CA, TCS-5\%P24/CA, and TCS-10\%P24/CA groups. Black arrow represents new vessel.

shape [28]. Tricalcium phosphate is similar to bone in chemical composition and crystal structure [35]. Due to its fine particle size and micropore structure, tricalcium phosphate has good bone conductivity and biodegradability. However, its application is limited by its brittleness, intolerance to pressure, and easy decomposition. Calcium sulfate is one of the earliest bone substitute materials; as a bone conduction mate- rial, it can be completely absorbed with the formation of bone tissue and bone structure remodeling, but it often reduces its mechanical strength because it is absorbed too fast, which is not conducive to the repair of bone defects at the weightbearing site [36]. Calcium phosphate cement is a kind of injectable biological bone substitute material, which has attracted many scholars in recent years because of its good 
biocompatibility and easy shaping. However, the material must be closely attached to the bone bed when filling the defect, and even so, its bone conductivity is still limited, which limits its clinical application [37].

Calcium carbonate is the main component of coral and widely used in the repair of bone defects in the clinic [22]. Calcium carbonate is bioabsorbable and has excellent biocompatibility and osteoconductivity. However, coral bone transplantation is confronting limitations, such as changes in physical marine, ecological environment, and chemical properties [38]. Synthetic calcium carbonatebased biomaterials are considered to be effective materials to replace corals to repair bone defects. The addition of carboxymethyl chitosan makes calcium carbonate hybrid microspheres have good biocompatibility, osteoconductivity, and biodegradability. The addition of carboxymethyl chitosan makes calcium carbonate hybrid microspheres have more excellent biocompatibility and biodegradability. Meanwhile, the potential application of this novel thiolated chitosan/calcium carbonate composite microsphere scaffold in bone tissue has not been studied.

Bone tissue engineering scaffolds usually require extremely porous and interconnected pore structures. More extensive pore size and porosity usually indicate a large surface area ratio, which is favorable toward cell adhesion to the scaffold and improves bone tissue reconstruction $[39,40]$. A previous study has shown that the growth quality of bones in porous systems depends on their pore size [41,42]. The optimal pore size for mineralized bone growth is still a controversial topic. The previous study has shown that the pore size of regenerated mineralized bone should be greater than $100 \mu \mathrm{m}$ [43]. A study [19] revealed pores greater than 20$100 \mu \mathrm{m}$ were beneficial to cell infiltration, and the neovascularization was improved significantly after exceeding $100 \mu \mathrm{m}$. The porous structure of our scaffold was obtained by a freeze-drying technique. The results of SEM demonstrated that the majority of the pores have a diameter from 90 $120 \mu \mathrm{m}$. Our studies further revealed that TCS-P24/CA scaffolds can effectively promote cell proliferation and bone growth, indicating that TCS-P24/CA scaffolds with a pore diameter of $90-120 \mu \mathrm{m}$ are suitable for bone growth.

In addition to the favorable controlled delivery performance of the TCS-P24/CA scaffolds, its biological effects are also desirable. It is well justified for their osteoconductivity and osteoinductivity of P24 peptides, P24 peptides play a significant role in osteogenic differentiation [44], they are indispensable in different stages of bone healing. A study [45] has revealed that adenovirus-mediated BMP therapy can cause harmful side effects, such as immunogenicity and tumorigenesis. However, biomaterials as carriers, which can provide controllable and continuous transmission of growth factors at the defect site and simulate its time distribution during bone healing in vivo [46]. The results showed that the TCS-P24/CA group maintains the prolonged release of P24 continued for 29 days, suggesting that the release velocity from the TCS-P24/CA scaffold manifests a sustained release of P24 and leads to osteogenic differentiation of the BMSCs and promoting bone regeneration.
The biological properties of the TCS-P24/CA scaffolds in vitro were investigated. CCK-8 analysis showed that the number of BMSCs in the TCS-P24/CA composite scaffolds group was similar to that in the control group, indicating that the composite stent provided a suitable microenvironment for cell proliferation. Furthermore, the important markers to assess the differentiation of osteoblasts are the activity of ALP and the level of calcium deposition and the expression of osteogenic genes. These results showed that ALP activity of TCS-10\%P24/CA group significantly increased on day 7 compared to the other groups. Similarly, the level of calcium mineral deposition increased as well in the TCS-10\%P24/CA group after 3 weeks. Furthermore, PCR results showed that Runx2, Alp, and COL1a1 mRNA expression increased significantly in the TCS-10\%P24/CA group. These results indicate that P24, when delivered in TCS-P24/CA scaffolds, still retains its biological ability. TCS-P24/CA scaffolds provide an effective approach to P24 delivery and have stimulatory effects on the matrix mineralization and differentiation of osteoblastic cells.

In vivo osteoinductive studies, to further evaluate the ability of TCS-P24/CA scaffolds and promote bone formation. We created calvarial bone defects in SD rats. These results demonstrated that BMD in the TCS-10\%P24/CA group was much higher than in the other groups and exhibited robust osteogenic activity. Histological analysis was similar to the BMD result (Figure 4). These data revealed that TCS-10\%P24/CA scaffolds enhanced the repair and mineralization of defects compared to other groups, indicating TCS-P24/CA that scaffolds are osteoconductive, while TCS-10\%P24/CA scaffold can provide an even more effective approach to the repair of a critical-sized bone defect. These results may be due to the role of TCS-P24/CA in promoting osteogenic differentiation in BMSCs and supporting BMSC adhesion and proliferation. These data confirmed the effectiveness and feasibility of developing functional TCSP24/CA scaffolds as a biological factor delivery platform for bone repair.

There are still some limitations in this experiment, such as the failure to establish more concentration gradients to explore the most appropriate concentration of P24 polypeptide to promote osteogenesis and further explore the signal transduction mechanism of materials in the process of osteogenesis. Thus, further experiments are needed to improve.

\section{Conclusions}

In this study, a novel thiolated chitosan-P24/calcium carbonate composite microspheres scaffold (TCS-P24/CA), synthesized by the in-situ coprecipitation and the freeze-drying methods, can steadily release P24 for 29 days. In vitro investigation regarding SEM showed that the majority of the pores have a diameter from $90-120 \mu \mathrm{m}$. Moreover, it was demonstrated to promote BMSCs adhesion, viability, and proliferation. Additionally, TCS-P24/CA induced osteogenic differentiation of BMSCs in vitro. Finally, the promoting effect of TCS-10\%P24/CA scaffold on bone regeneration was significantly higher than that of other groups in vivo. The novel TCS-P24/CA scaffold is expected to become a 
novel bone substitute material with clinical application prospects.

\section{Data Availability}

The data used to support the findings of this study are included within the article.

\section{Conflicts of Interest}

The authors declare that they have no conflicts of interest.

\section{Authors' Contributions}

Zhaozhen Wang, Xujie Liu, and Vidmi Taolam Martin contributed equally to this work.

\section{Acknowledgments}

Financial support from the Basic Research on Scientific creation Committee of Shenzhen City, China (JCYJ20160 531195524566) is gratefully acknowledged.

\section{References}

[1] L. Li, G. Zhou, Y. Wang, G. Yang, S. Ding, and S. Zhou, "Controlled dual delivery of BMP-2 and dexamethasone by nanoparticle-embedded electrospun nanofibers for the efficient repair of critical-sized rat calvarial defect," Biomaterials, vol. 37, pp. 218-229, 2015.

[2] Y.Li, Z. Zhang, and Z. Zhang, "Porous chitosan/nano-hydroxyapatite composite scaffolds incorporating simvastatin-loaded PLGA microspheres for bone repair," Cells, Tissues, Organs, vol. 205, no. 1, pp. 20-31, 2018.

[3] Y. Chen, X. Liu, R. Liu et al., "Zero-order controlled release of BMP2-derived peptide P24 from the chitosan scaffold by chemical grafting modification technique for promotion of osteogenesisinvitro and enhancement of bone repairin vivo," Theranostics, vol. 7, no. 5, pp. 1072-1087, 2017.

[4] L. Ning, H. Malmström, and Y. F. Ren, "Porous collagenhydroxyapatite scaffolds with mesenchymal stem cells for bone regeneration," The Journal of Oral Implantology, vol. 41, no. 1, pp. 45-49, 2015.

[5] R. Hou, F. Chen, Y. Yang et al., "Comparative study between coral-mesenchymal stem cells-rhBMP-2 composite and autobone-graft in rabbit critical-sized cranial defect model," Journal of Biomedical Materials Research Part A, vol. 80, no. 1, pp. 85-93, 2007.

[6] Y. Gong, S. Li, W. Zeng, J. Yu, Y. Chen, and B. Yu, "Controlled In vivo bone formation and vascularization using ultrasoundtriggered release of recombinant vascular endothelial growth factor from Poly(D,L-lactic-co-glycolicacid) microbubbles," Frontiers in Pharmacology, vol. 10, p. 413, 2019.

[7] X. Liu, Q. Feng, A. Bachhuka, and K. Vasilev, "Surface modification by allylamine plasma polymerization promotes osteogenic differentiation of human adipose-derived stem cells," ACS Applied Materials \& Interfaces, vol. 6, no. 12, pp. 97339741, 2014.

[8] I. H. Bae, B. C. Jeong, M. S. Kook, S. H. Kim, and J. T. Koh, "Evaluation of a thiolated chitosan scaffold for local delivery of BMP-2 for osteogenic differentiation and ectopic bone for- mation," BioMed Research International, vol. 2013, Article ID 878930, 10 pages, 2013.

[9] S. D. Nath, C. Abueva, B. Kim, and B. T. Lee, "Chitosan-hyaluronic acid polyelectrolyte complex scaffold crosslinked with genipin for immobilization and controlled release of BMP2," Carbohydrate Polymers, vol. 115, pp. 160-169, 2015.

[10] S. E. Kim, Y. P. Yun, Y. K. Han et al., "Osteogenesis induction of periodontal ligament cells onto bone morphogenic protein2 immobilized PCL fibers," Carbohydrate Polymers, vol. 99, pp. 700-709, 2014.

[11] M. Raida, A. C. Heymann, C. Günther, and D. Niederwieser, "Role of bone morphogenetic protein 2 in the crosstalk between endothelial progenitor cells and mesenchymal stem cells," International Journal of Molecular Medicine, vol. 18, no. 4, pp. 735-739, 2006.

[12] H. Kaneko, T. Arakawa, H. Mano et al., "Direct stimulation of osteoclastic bone resorption by bone morphogenetic protein (BMP)-2 and expression of BMP receptors in mature osteoclasts," Bone, vol. 27, no. 4, pp. 479-486, 2000.

[13] A. Saito, Y. Suzuki, S. I. Ogata, C. Ohtsuki, and M. Tanihara, "Activation of osteo-progenitor cells by a novel synthetic peptide derived from the bone morphogenetic protein-2 knuckle epitope," Biochim Biophys Acta, vol. 1651, no. 1-2, pp. 60-67, 2003.

[14] B. Wu, Q. Zheng, X. Guo, Y. Wu, Y. Wang, and F. Cui, “Preparation and ectopic osteogenesis in vivo of scaffold based on mineralized recombinant human-like collagen loaded with synthetic BMP-2-derived peptide," Biomedical Materials (Bristol, England), vol. 3, no. 4, article 044111, 2008.

[15] Y. Boukari, O. Qutachi, D. J. Scurr, A. P. Morris, S. W. Doughty, and N. Billa, "A dual-application poly (dl-lacticco-glycolic) acid (PLGA)-chitosan composite scaffold for potential use in bone tissue engineering," Journal of Biomaterials Science Polymer Edition, vol. 28, no. 16, pp. 1966-1983, 2017.

[16] K. Kafedjiiski, A. H. Krauland, M. H. Hoffer, and A. BernkopSchnürch, "Synthesis and in vitro evaluation of a novel thiolated chitosan," Biomaterials, vol. 26, no. 7, pp. 819-826, 2005.

[17] A. Matsuda, H. Kobayashi, S. Itoh, K. Kataoka, and J. Tanaka, "Immobilization of laminin peptide in molecularly aligned chitosan by covalent bonding," Biomaterials, vol. 26, no. 15, pp. 2273-2279, 2005.

[18] X. Liu, B. Yu, Q. Huang et al., "In vitro BMP-2 peptide release from thiolated chitosan based hydrogel," International Journal of Biological Macromolecules, vol. 93, Part A, pp. 314-321, 2016.

[19] X. He, Y. Liu, X. Yuan, and L. Lu, "Enhanced healing of rat calvarial defects with MSCs loaded on BMP-2 releasing chitosan/alginate/hydroxyapatite scaffolds," PLoS One, vol. 9, no. 8, article e104061, 2014.

[20] H. D. Yu, Z. Y. Zhang, K. Y. Win, J. Chan, S. H. Teoh, and M. Y. Han, "Bioinspired fabrication of 3D hierarchical porous nanomicrostructures of calcium carbonate for bone regeneration," Chemical Communications (Cambridge, England), vol. 46, no. 35, pp. 6578-6580, 2010.

[21] H.-C. Ge and D.-K. Luo, "Preparation of carboxymethyl chitosan in aqueous solution under microwave irradiation," Carbohydrate Research, vol. 340, no. 7, pp. 1351-1356, 2005.

[22] F. He, J. Zhang, X. Tian, S. Wu, and X. Chen, "A facile magnesium-containing calcium carbonate biomaterial as 
potential bone graft," Colloids and Surfaces B: Biointerfaces, vol. 136, pp. 845-852, 2015.

[23] J. Wang, J. S. Chen, J. Y. Zong et al., "Calcium carbonate/carboxymethyl chitosan hybrid microspheres and nanospheres for drug delivery," The Journal of Physical Chemistry C, vol. 114, no. 44, pp. 18940-18945, 2010.

[24] F. He, J. Zhang, F. Yang, J. Zhu, X. Tian, and X. Chen, "In vitro degradation and cell response of calcium carbonate composite ceramic in comparison with other synthetic bone substitute materials," Materials Science \& Engineering C: Materials for Biological Applications, vol. 50, pp. 257-265, 2015.

[25] X. Liu, T. A. Elkhooly, Q. Huang et al., “A facile way to prepare mesoporous spherical calcites controlled by chondroitin sulfate for shape and carboxymethyl chitosan for size," CrystEngComm, vol. 18, no. 44, pp. 8582-8586, 2016.

[26] X. Liu, Y. Chen, Q. Huang, W. He, Q. Feng, and B. Yu, “A novel thermo-sensitive hydrogel based on thiolated chitosan/hydroxyapatite/beta-glycerophosphate," Carbohydrate Polymers, vol. 110, pp. 62-69, 2014.

[27] B. Yu, Y. Zhang, X. Li et al., "The use of injectable chitosan/nanohydroxyapatite/collagen composites with bone marrow mesenchymal stem cells to promote ectopic bone formation in vivo," Journal of Nanomaterials, vol. 2013, Article ID 506593, 8 pages, 2013.

[28] Y. Chen, X. Liu, R. Liu et al., "Zero-order controlled release of BMP2-derived peptide P24 from the chitosan scaffold by chemical grafting modification technique for promotion of osteogenesis in vitro and enhancement of bone repair in vivo," Theranostics, vol. 7, no. 5, pp. 1072-1087, 2017.

[29] T. Garg, O. Singh, S. Arora, and R. S. R. Murthy, "Scaffold: a novel carrier for cell and drug delivery," Critical Reviews in Therapeutic Drug Carrier Systems, vol. 29, no. 1, pp. 1-63, 2012.

[30] X. Li, J. Wei, K. E. Aifantis et al., "Current investigations into magnetic nanoparticles for biomedical applications," Journal of Biomedical Materials Research Part A, vol. 104, no. 5, pp. 1285-1296, 2016.

[31] W.-G. La, S.-W. Kang, H. S. Yang et al., "The efficacy of bone morphogenetic protein-2 depends on its mode of delivery," Artificial Organs, vol. 34, no. 12, pp. 1150-1153, 2010.

[32] K. J. Rambhia and P. X. Ma, "Controlled drug release for tissue engineering," Journal of Controlled Release: Official Journal of the Controlled Release Society, vol. 219, pp. 119-128, 2015.

[33] L. Yin, J. Ding, C. He, L. Cui, C. Tang, and C. Yin, "Drug permeability and mucoadhesion properties of thiolated trimethyl chitosan nanoparticles in oral insulin delivery," Biomaterials, vol. 30, no. 29, pp. 5691-5700, 2009.

[34] S. K. Nandi, S. Roy, P. Mukherjee, B. Kundu, D. K. de, and D. Basu, "Orthopaedic applications of bone graft \&amp; graft substitutes: a review," The Indian Journal of Medical Research, vol. 132, pp. 15-30, 2010.

[35] S. K. Ghosh, S. K. Nandi, B. Kundu et al., "In vivo response of porous hydroxyapatite and $\beta$-tricalcium phosphate prepared by aqueous solution combustion method and comparison with bioglass scaffolds," Journal of Biomedical Materials Research Part B: Applied Biomaterials, vol. 86B, no. 1, pp. 217-227, 2008.

[36] K. A. Hing, L. F. Wilson, and T. Buckland, "Comparative performance of three ceramic bone graft substitutes," The Spine Journal, vol. 7, no. 4, pp. 475-490, 2007.
[37] C. Knabe, G. Berger, R. Gildenhaar et al., "Effect of rapidly resorbable calcium phosphates and a calcium phosphate bone cement on the expression of bone-related genes and proteinsin vitro," Journal of Biomedical Materials Research, vol. 69A, no. 1, pp. 145-154, 2004.

[38] S. A. Clarke, P. Walsh, C. A. Maggs, and F. Buchanan, "Designs from the deep: marine organisms for bone tissue engineering," Biotechnology Advances, vol. 29, no. 6, pp. 610617, 2011.

[39] A. Cipitria, C. Lange, H. Schell et al., "Porous scaffold architecture guides tissue formation," Journal of Bone and Mineral Research: The Official Journal of the American Society for Bone and Mineral Research, vol. 27, no. 6, pp. 1275-1288, 2012.

[40] K. Zhang, Y. Fan, N. Dunne, and X. Li, "Effect of microporosity on scaffolds for bone tissue engineering," Regenerative Biomaterials, vol. 5, no. 2, pp. 115-124, 2018.

[41] A. I. Itälä, H. O. Ylänen, C. Ekholm, K. H. Karlsson, and H. T. Aro, "Pore diameter of more than 100 microm is not requisite for bone ingrowth in rabbits," Journal of Biomedical Materials Research, vol. 58, no. 6, pp. 679-683, 2001.

[42] A. Aarvold, J. O. Smith, E. R. Tayton et al., "The effect of porosity of a biphasic ceramic scaffold on human skeletal stem cell growth and differentiation in vivo," Journal of Biomedical Materials Research Part A, vol. 101, no. 12, pp. 3431-3437, 2013.

[43] S. F. Hulbert, F. A. Young, R. S. Mathews, J. J. Klawitter, C. D. Talbert, and F. H. Stelling, "Potential of ceramic materials as permanently implantable skeletal prostheses," Journal of Biomedical Materials Research, vol. 4, no. 3, pp. 433-456, 1970.

[44] H. Liu, H. Peng, Y. Wu et al., "The promotion of bone regeneration by nanofibrous hydroxyapatite/chitosan scaffolds by effects on integrin-BMP/Smad signaling pathway in BMSCs," Biomaterials, vol. 34, no. 18, pp. 4404-4417, 2013.

[45] Y. Liu, S. Zhang, G. Ma, F. Zhang, and R. Hu, "Efficacy and safety of a live canine adenovirus-vectored rabies virus vaccine in swine," Vaccine, vol. 26, no. 42, pp. 5368-5372, 2008.

[46] M. Yamamoto, Y. Takahashi, and Y. Tabata, "Controlled release by biodegradable hydrogels enhances the ectopic bone formation of bone morphogenetic protein," Biomaterials, vol. 24, no. 24, pp. 4375-4383, 2003. 